\title{
Deciphering the Different Types of Refractory Celiac Disease
}

\author{
Georgia Malamut $^{1,2,3,}$, Nadine Cerf-Bensussan ${ }^{1,3}$, Christophe Cellier ${ }^{1,2,3}$ \\ ${ }^{1}$ Université Paris Descartes-Sorbonne Paris Centre \\ ${ }^{2}$ Gastroenterology department, Hôpital Européen Georges Pompidou APHP, Paris \\ ${ }^{3}$ Inserm U989, Hôpital Européen Georges Pompidou APHP Paris, France
}

Fifteen years ago has emerged the concept of refractory celiac disease (RCD). [1,2] RCD is defined by persisting malabsorption and villous atrophy after one year of strict GFD ascertained by a dietician. RCD has been subdivided into two subgroups according to the normal (type I RCD (RCDI)) or abnormal phenotype of intraepithelial lymphocytes (IEL) (type II RCD (RCDII). RCDI is indistinguishable from active $\mathrm{CD}$ and is characterized by a polyclonal repertoire. Survival of patients with RCDI is slightly inferior to that of those with celiac disease. [3,4] In contrast, RCDII is a severe enteropathy with frequent intractable ulcerative duodeno-jejunitis. RCDII is characterized by a clonal repertoire and is considered as a low-grade intraepithelial lymphoma. [1,2] The five year survival rate of patients with RCDII is around 50\%. This poor prognosis is due to severe malnutrition and increased risk of overt lymphoma.

One of the current challenges in RCD is to ascertain the type of RCD. Indeed, diagnosis of RCD has become highly specialized. It requires small bowel endoscopic specialized investigations such as capsule endoscopy and double balloon enteroscopy. [5,6] Intestinal biopsy needs to be studied by different complementary techniques such as immunohistochemistry, flow cytometry, and Multiplex PCR, all necessary to ascertain the precise diagnosis. Recent studies have reported case series of chronic intestinal diseases mimicking the both types of RCD. RCDI needs to be distinguished from other causes of non clonal villous atrophy such as enteropathy associated with common variable immunodeficiency (CVID). [7] Demonstration of HLA haplotypes encoding HLA-DQ2 or DQ8 can be useful as their absence excludes CD or RCD as a cause of villous atrophy. [8] Nevertheless, genotypes HLA-DQ2/DQ8 are also found in 77\% of CVID patients with enteropathy mimicking CD defined by intestinal intra-epithelial lymphocytosis. [7] So evidence of serum hypogammaglobulinemia and histopathological features such as intestinal plasmocytic rarefaction or nodular lymphoid hyperplasia appear essential to distinct CVID enteropathy from RCDI. [7] RCDII needs to be distinguished from other cases of clonal enteropathy with villous atrophy. Flow cytometry allows to tell the difference between RCDII and other intestinal clonal malignancies with villous atrophy. Indeed, demonstration of excess of CD4+IEL with a specific Vbeta repertoire is essential for diagnosis of intestinal CD4 lymphoproliferations. [9] Diagnosis may be particularly tricky when CD4 lymphoproliferation [9] or large granular lymphocytic leukemia (LGL) [10] complicate an authentic
CD. Consequently, spectrum of RCD has been recently substantially extended. Advances in accuracy of diagnostic tools could reduce the number of undiagnosed RCD and increase the frequency of RCD currently estimated to 1 to $2 \%$ of CD patients. [11,12] Besides epidemiological interest, improvement of diagnostic tools allowed precise diagnosis of RCD type and appropriate treatment. By example, distinction between RCDII and CD complicated by LGL is crucial as cyclosporin, inefficient in RCDII, is in contrast very useful for treating LGL. Indeed, treatment of RCD remains another challenge particularly in case of RCDII patients who have poor prognosis and for whom referral treatment does not yet exist. Immunosuppressors are poorly efficient and may possibly trigger overt lymphoma. [13] Purine analogues such pentostatine or cladribine (2 CDA) have been largely used in the past with slight therapeutic effect. $[4,14,15]$ Autologous haematopoietic stem cells transplantation represents an interesting alternative but when combined to chemotherapy for hopping sustained reduction of abnormal IEL. [16,17] We are currently evaluating this strategy in a prospective phase II trial. Many advances in the understanding of the pathogenesis of RCDII were made by deciphering the anti-apoptotic signaling pathway of the cytokine IL-15 which prevents the elimination of IEL in CD and RCDII. [18] The IL-15 induced antiapoptotic signaling pathway includes activation of IL15R $\beta \gamma$, Jak3, STAT5 and Bcl-xL [19] which represent so many therapeutic targets. Blocking the effect of IL-15 appears of a particular interest in RCDII but may probably be useful in other types of RCD with possible involvement of IL-15 such as RCDI or LGL-complicated CD. [20] Continuing the characterization of the different forms of RCD will probably guide next pathogenic studies and permit to increase our therapeutic efficacy in the future.

\section{References}

[1] Cellier C, Patey N, Mauvieux L, et al. Abnormal intestinal intraepithelial lymphocytes in refractory sprue. Gastroenterology 1998; 114: 471-81.

[2] Cellier C, Delabesse E, Helmer C, et al. Refractory sprue, coeliac disease, and enteropathy-associated T-cell lymphoma. French Coeliac Disease Study Group. Lancet 2000; 356: 203-8.

[3] Al-Toma A, Verbeek WH, Hadithi M, et al. Survival in Refractory Coeliac Disease and Enteropathy associated T cell Lymphoma: Retrospective evaluation of single centre experience. Gut 2007; 56: 1373-8.

[4] Malamut G, Afchain P, Verkarre V, et al. Presentation and longterm follow-up of refractory celiac disease: comparison of type I with type II. Gastroenterology 2009; 136: 81-90. 
[5] Daum S, Wahnschaffe U, Glasenapp R, et al. Capsule endoscopy in refractory celiac disease. Endoscopy 2007; 39: 455-8.

[6] Barret M, Malamut G, Rahmi G, et al. Diagnostic yield of capsule endoscopy in refractory celiac disease. Am J Gastroenterol 2012; 107: 1546-53.

[7] Malamut G, Verkarre V, Suarez F, et al. The enteropathy associated with common variable immunodeficiency: the delineated frontiers with celiac disease. The American journal of gastroenterology 2010; 105: 2262-75.

[8] Green PH, Cellier C. Celiac disease. The New England journal of medicine 2007; 357: 1731-43.

[9] Malamut G, Meresse B, Kaltenbach S, et al. Small Intestinal CD4+ T-cell Lymphoma is a Heterogenous Entity with Common Pathology Features. Clin Gastroenterol Hepatol 2013. Clin Dec 3. [Epub ahead of print].

[10] Malamut G, Meresse B, Verkarre V, et al. Large granular lymphocytic leukemia: a treatable form of refractory celiac disease. Gastroenterology 2012;143:1470-2.

[11] West J. Celiac disease and its complications: a time traveller's perspective. Gastroenterology 2009; 136: 32-4.

[12] Roshan B, Leffler DA, Jamma S, et al. The incidence and clinical spectrum of refractory celiac disease in a north american referral center. The American journal of gastroenterology 2011; 106: 9238.
[13] Goerres MS, Meijer JW, Wahab PJ, et al. Azathioprine and prednisone combination therapy in refractory coeliac disease. Aliment Pharmacol Ther 2003; 18: 487-94.

[14] Dray X, Joly F, Lavergne-Slove A, et al. A severe but reversible refractory sprue. Gut 2006; 55: 1210-1.

[15] Al-Toma A, Goerres MS, Meijer JW, et al. Cladribine therapy in refractory celiac disease with aberrant T cells. Clin Gastroenterol Hepatol 2006; 4: 1322-7.

[16] Al-Toma A, Visser OJ, van Roessel HM, et al. Autologous hematopoietic stem cell transplantation in refractory celiac disease with aberrant T-cells. Blood. 2007; 109(5): 2243-9.

[17] Tack GJ, Wondergem MJ, Al-Toma A, et al. Auto-SCT in refractory celiac disease type II patients unresponsive to cladribine therapy. Bone marrow transplantation; 46: 840-6.

[18] Mention JJ, Ben Ahmed M, Begue B, et al. Interleukin 15: a key to disrupted intraepithelial lymphocyte homeostasis and lymphomagenesis in celiac disease. Gastroenterology 2003; 125: $730-45$.

[19] Malamut G, El Machhour R, Montcuquet N, et al. IL-15 triggers an antiapoptotic pathway in human intraepithelial lymphocytes that is a potential new target in celiac disease-associated inflammation and lymphomagenesis. The Journal of clinical investigation 2010; 120: 2131-43.

[20] Chen J, Petrus M, Bamford R, et al. Increased serum soluble interleukin-15 receptor alpha (sIL-15Ralpha) levels in T-cell large granular lymphocyte leukemia. Blood. 2012 Jan 5; 119(1): 137-43. 